\title{
Type 2 Diabetes-Driven Alterations in Bone Healing and Angiogenesis
}

\author{
Seungyup Sun ${ }^{1}$, Fazal Ur Rehman Bhatti ${ }^{1,2}$, Ushashi C. Dadwal ${ }^{1,2}$, Deepa Sheik \\ Pran Babu ${ }^{1,2}$, Anthony J. Perugini III ${ }^{1}$, Olatundun D. Awosanya ${ }^{1}$, Rachel J. \\ Blosser $^{1,2}$, Sarah A. Tersey ${ }^{1}$, Kara S. Orr ${ }^{1}$, Karishma R. Randhave ${ }^{1}$, Jiliang $\mathrm{Li}^{3}$, \\ Mervin C. Yoder ${ }^{1}$, Carmella Evans-Molina ${ }^{1,2}$, and Melissa A. Kacena ${ }^{1,2}$ \\ ${ }^{1}$ Indiana University School of Medicine, Indiana, USA; ${ }^{2}$ Richard L. Roudebush VA \\ Medical Center, Indiana, USA; ${ }^{3}$ Indiana University - Purdue University \\ Indianapolis, Indiana, USA
}

Background and Hypothesis: Type 2 diabetes (T2D) is prevalent in the United States. T2D patients are at risk for impaired fracture healing due to decreased angiogenesis, which is required for successful bone regeneration. Bone morphogenetic protein-2 (BMP-2) is often used to help orthopedic surgeons with bone healing in difficult cases. Here, we begin characterizing the mechanism by which T2D alters bone healing with or without BMP-2 treatment. We hypothesize that T2D impairs fracture healing by decreasing angiogenesis and endothelial cell function.

Project Methods: Using Tie2-CreER;Td-Tomato mice (Tie2 $2^{\mathrm{CreERT+}}$ ), we established a high fat diet (HFD)-induced T2D mouse model to compare with control low fat diet (LFD)-fed mice. Mice underwent testing to confirm the T2D-like metabolic phenotype, underwent a femoral critical-size defect surgery that was treated with either saline or BMP-2, and were then assessed biweekly by X-ray imaging over the course of 12 weeks. Finally, bone marrow-derived endothelial cells were collected from these mice to assess changes in endothelial colony and tube formation in vitro.

Results: Results showed that the HFD mice acquired the T2D metabolic phenotype. Fracture healing was impaired in the HFD mice, even with BMP-2 treatment. The isolation of BMECs was confirmed by visualization of fluorescent Tie2+ cells. Unexpectedly, in vitro tube formation assays indicated that HFD improved vessel-like formation properties. BMP-2 treatment appeared to improve some vessel-like formation properties compared to control treatment.

Conclusion and Potential Impact: This study is ongoing. Further data will need to be collected to better characterize differences in bone healing and angiogenesis in the healing femurs. Still, these data reveal the mechanisms by which T2D impairs bone healing and demonstrate the important difference between examining endothelial cells in vitro vs. in vivo. Future investigations will examine if thrombopoietin, which our group has previously shown to improve both fracture healing and angiogenesis, may be a more effective treatment than BMP-2 in this model. 